\title{
Nanotechnology for Materials and Manufacturing - Physics, Synthesis, and Devices
}

Qing Hao*

DOI: $10.30919 / \mathrm{esmm} 5 \mathrm{f} 322$

Nanotechnology introduces unprecedented research opportunities in various energy and environment related applications. This issue in ES Materials and Manufacturing contains many interesting papers to show the potential development along this important research direction, ranging from fundamental physics, materials synthesis, to novel device applications.

As the most ubiquitous form of energy, heat has been used ever since the beginning of human being history and thermal sciences still remain as a vibrant field with new possibility in nanomaterials. In this issue, Xiao et al. (10.30919/esmm5f237) reviewed the recent studies on thermal transport within periodic porous films, 2D materials, and 3D structures. These materials have been widely studied in recent years for potential applications in thermoelectric energy conversion and thermal management. When heat can be manipulated as waves instead of particlelike phonons, existing studies on acoustic/optics can be extended to thermal applications. This review mainly addresses the challenge and limitation for such "phononic crystals," in analogy to "photonic crystals" for light manipulation. Other than material development, new measurement techniques are also critical to thermal studies. Wang et al. (10.30919/esmm5f239) proposed a modified steady-state hot wire method that can characterize convection heat transfer coefficient of microwires without the knowledge of thermal conductivity for the microwire. This technique can be potentially used for microscale to nanoscale convection studies.

For mass production of materials in manufacturing, enormous attention should be paid to the process-structure-property relationships due to employed synthesis techniques. Here Mitkari and Ubale (10.30919/esmm5f231) grew nanostructured CoS thin films on an amorphous glass substrate by the SILAR method. These films showed

Department of Aerospace and Mechanical Engineering, University of Arizona, Tucson, AZ 85721 U.S.A

*E-mail: qinghao@email.arizona.edu hexagonal structures, while their electrical resistivity and activation energy were found to be thickness dependent. For thermoelectrics, Artini et al. (10.30919/esmm5f221) varied the applied pressure used for the spark plasma sintering of filled skutterudite. The resulting power factors were checked for samples belonging to the $\mathrm{Sm}_{\mathrm{y}}\left(\mathrm{Fe}_{\mathrm{x}} \mathrm{Ni}_{1-\mathrm{x}}\right)_{4} \mathrm{Sb}_{12}$ system. Yu and Sun reported a facile one-pot synthesis method to prepare well dispersed silver nanoparticles supported on $\alpha$-zirconium phosphate single-layer nanosheets. Fundamentally, Shen et al. (10.30919/esmm5f233) measured the solubility of tylosin in pure solvents and acetone + water mixture solvents, with comparison to thermodynamics models.

Important applications of new materials are often related to novel devices. For broadband photo-detection applications, Jadkar et al. (10.30919/esmm5f236) synthesized highly crystalline hydrogenated nanocrystalline silicon thin films by the hot wire method. Ghafari et al. developed an efficient piezoelectric nanogenerator using polyvinylidene fluoride polymers to harvest the mechanical energy in the low-frequency range. More broadly, the development of nanotechnology can also benefit conventional fields such as agriculture. Vassell and Mao (10.30919/esmm5f212) studied the enhanced quality and growth of kale (Brassica oleracea) plant with copper oxide nanoparticles to coat kale seeds.

In summary, the rapid development of materials science and nanophysics has opened many interesting research directions. In the future, more efforts should be dedicated to both the fundamental and practical sides to provide low-cost and high-performance materials and devices. We sincerely hope the readers can feel the excitement for this collection of papers.

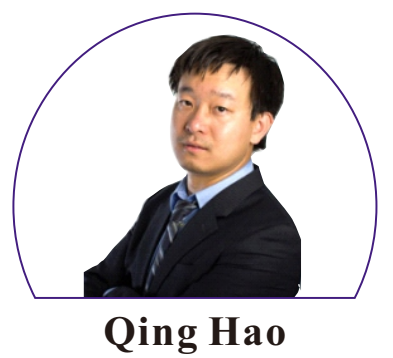

Qing Hao is an Associate Professor of Department of Aerospace and Mechanical Engineering at the University of Arizona. His research focuses on nanoscale energy transport in materials and electronic devices, including thermoelectrics, high-power electronics, and $2 D$ materials. He received the $2008 R \& D 100$ Award as a team member for thermoelectrics research, 2015 AFOSR YIP Award for graphene studies, and 2017 NSF CAREER Award for thermal studies of grain boundaries. 